SCIENTIFIC REPORT

\title{
Visual loss following removal of intraocular silicone oil
}

\section{S Cazabon, C Groenewald, I A Pearce, D Wong}

Br J Ophthalmol 2005;89:799-802. doi: 10.1136/bjo.2004.053561

\begin{abstract}
Aim: To investigate the cause of visual loss following removal of intraocular silicone oil in patients who underwent vitrectomies for retinal detachment or giant retinal tear. Methods: The clinical records of three cases with visual loss following removal of silicone oil were reviewed. Investigations carried out included fundus fluorescein angiogram, optical coherence tomography, and electrophysiological studies.

Results: Visual acuities dropped from $6 / 9$ to $6 / 36$ in two cases and $6 / 24$ to $3 / 24$ in the third. None of the three cases had macula detachment at any stage. Fundus fluorescein angiogram and optical coherence tomography were normal in all cases. Pattern electroretinogram showed reduced amplitudes of the P50 and N95 components. Multifocal electroretinogram indicated a selective damage to the central part of the macula.

Conclusions: The results suggest that the abnormality arises predominantly in the central part of the macula, especially the outer and middle layers. However, the exact mechanism still remains obscure.
\end{abstract}

$\mathrm{S}$ ilicone oil has a well established role in vitreoretinal surgery. With the improvement of microsurgical techniques, silicone oil has been successfully used in the management of complicated retinal detachments associated with proliferative vitreoretinopathy, giant retinal tears, proliferative diabetic retinopathy, severe trauma, and cytomegalovirus related retinal detachments. ${ }^{1-5}$ It provides prolonged intraocular tamponade and because of its optical clarity, it allows for adequate postoperative assessment.

"Unexplained" visual loss is a rare complication following oil removal. It has hitherto not been reported in the literature to the best of our knowledge, although oral case presentations have been made at successive meetings of the British and Eire Association of Vitreoretinal Surgeons (BEAVRS). We recently encountered three cases and decided to carry out special investigations to elucidate the nature and cause of the visual loss.

\section{MATERIALS AND METHODS}

We reviewed the clinical records of three cases with visual loss following removal of intraocular silicone oil 5700 centistokes (Oxane 5700, Bauch \& Lomb). All three patients underwent uncomplicated pars plana vitrectomy and silicone oil injection for retinal detachments or giant retinal tear. Hartman's solution was used in exchange at the time of oil removal in each case. All three patients had optical coherence tomography (OCT) measurements of the central macula postoperatively. The OCT 3000 (Zeiss-Humphrey, USA) was used for the topographic mapping.

Horizontal line scans through the fovea of default length $5 \mathrm{~mm}$ at $0^{\circ}$ and a fast macula thickness map consisting of six $6 \mathrm{~mm}$ radial line scans centred on fixation were obtained.
Full field electroretinograms (ERGs) were obtained following the International Society of Clinical Electrophysiology of Vision (ISCEV) standards. Multifocal electroretinogram (mfERG) recorded binocularly with a stimulus size of 61 hexagons using the RETI scan system (Roland Consult, Wiesbaden, Germany) was used to assess macula function. Pupils were dilated and refractive errors were corrected in each case. The stimulus was at a distance of $380 \mathrm{~mm}$ from the patient's eye so that a field of 20 degree radius in 61 segments was stimulated. The patient fixated on the centre of a large diagonal cross, centred at the central hexagon. Each recording session consisted of eight trials over approximately 15 minutes. All three patients are still attending our clinic. Patients 1 and 2 have been attending for at least 2 years and patient 3 for 1 year.

\section{CASE 1}

A 45 year old myopic male patient presented with a 1 week history of floaters in his right eye. He was otherwise fit and healthy. On examination, his corrected visual acuity was right eye $6 / 5$, left eye $6 / 5$. The anterior segment was normal in both eyes. Funduscopy of his right eye showed a giant retinal tear in the nasal aspect involving about 5 clock hours. The disc was normal and the macula was attached. He underwent an uncomplicated three port pars plana vitrectomy and silicone oil injection under local anaesthetic the following morning. One week later his visual acuity was $6 / 9^{+3}$ in the right eye and the IOP was $23 \mathrm{~mm} \mathrm{Hg}$. At the 3 month postoperative visit, the visual acuity dropped to $6 / 12$ but he was noted to have a posterior subcapsular cataract.

One month later the silicone oil was removed, combined with phacoemulsification and intraocular lens (IOL) implantation under local anaesthetic. The surgery was uneventful, but a few days afterwards he complained of seeing a black shadow in the central aspect of his right eye. The unaided visual acuity was $1 / 60$ improving to $6 / 36$ on refraction. The anterior segment was normal and the IOP was $18 \mathrm{~mm} \mathrm{Hg}$ in his right eye. Funduscopy revealed a healthy disc and normal looking macula with a flat retina. Fundus fluorescein angiogram (FFA) and OCT did not reveal any abnormalities.

Pattern electroretinogram (PERG) was normal in both eyes but the right eye had smaller responses than the left eye. Both the P50 and N95 amplitudes were reduced in the right eye (see table 1). Standard flash ERG in light background revealed delayed latency in the right eye compared to the left. The oscillatory potential also showed reduced amplitude in the right eye compared to the left eye. MfERG indicated a well define hill of vision in the left eye but a more plateau effect in the right eye (see figs 1 and 2). The flash visual

Abbreviations: FFA, fundus fluorescein angiogram; IOL, intraocular lens; mfERG, multifocal electroretinogram; OCT, optical coherence tomography; PERG, pattern electroretinogram; VEP, visual evoked potential 

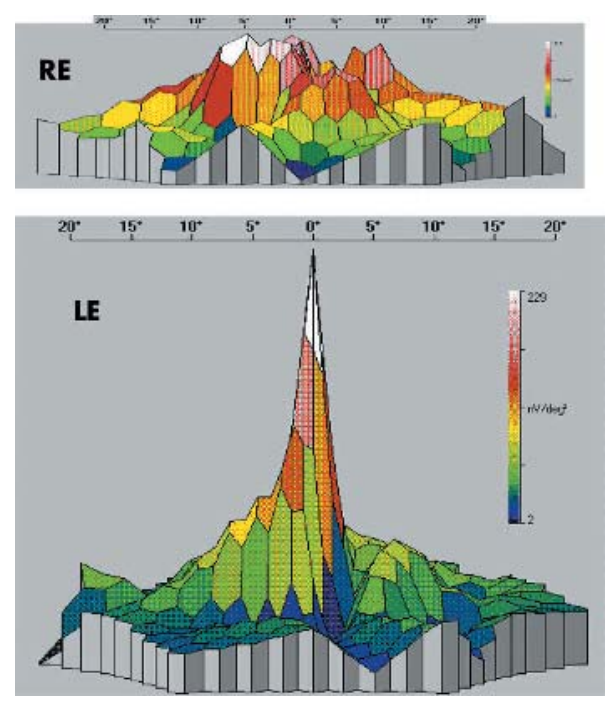

Figure 1 Multifocal ERG responses comparing affected right eye with unaffected left eye: case 1.

evoked potential (VEP) showed normal responses in both eyes.

\section{CASE 2}

A 55 year old myopic male presented with a 2 day history of reduced vision and floaters in his right eye. His visual acuity was $6 / 60$ in the right eye and 6/5 in the left eye. The anterior segment was normal but he had a moderately dense vitreous haemorrhage in his right eye. Funduscopy revealed a superior bullous macula on retinal detachment and lattice degeneration with three tears superiorly and two inferiorly. He underwent a three port pars plana vitrectomy and silicone oil injection under local anaesthetic on the same day. The following day his visual acuity improved to $6 / 9^{+3}$ in the right eye. The IOP was $20 \mathrm{~mm} \mathrm{Hg}$ and funduscopy showed a flat retina. About 5 months later, the silicone oil was removed, combined with phacoemulsification and IOL implantation under local anaesthetic because of the formation of a cataract.

One week after surgery he complained of blurred vision in his right eye. The IOP was normal and funduscopy showed a flat retina with a normal looking disc and macula. His best corrected visual acuity was 6/36 in the right eye. OCT of his right eye was normal.

The amplitudes of the P50 and N95 waves were small in both eyes, but even smaller in the affected eye compared to the unaffected eye (see table 1). ERG tests including standard and dim flash in the dark and white flash in light background produced smaller amplitudes and delayed responses in the right eye compared to the left eye. Multifocal ERG again indicated reduced central macula function in the right eye. The flash VEP was obtained and the responses were normal in both eyes.

\section{CASE 3}

A 42 year old myopic male complained of flashing lights and seeing a shadow in the lateral aspect of his left eye for 2 days. His left eye was known to be amblyopic. On examination his corrected visual acuity was $6 / 9$ in the right eye and 6/18 in the left. Funduscopy of his left eye showed a bulbous detachment involving the nasal half of the retina with two large breaks. The macula was not involved. The next day he underwent an uncomplicated three port pars plana vitrectomy, cryotherapy to the breaks, and silicone oil injection under general anaesthesia. His visual acuity with the silicone oil in the left eye was $6 / 24$ at 1 week postoperatively. The silicone oil was removed under a local anaesthetic 3 months after the vitrectomy.

One week later his visual acuity was reduced to $1 / 60$ in the left eye. Clinical examination did not reveal any abnormal findings to account for his reduced vision. The retina was flat and the optic disc and macula appeared normal. His best corrected visual acuity was 3/24 in the left eye. OCT of his left eye did not reveal any abnormalities.

Pattern ERG showed markedly reduced amplitudes of the P50 and N95 as well as increase latencies in the left eye (see table 1). Responses in the right eye were normal. Photopic ERG revealed smaller amplitudes and delayed latency from the left eye to both standard flash and $30 \mathrm{~Hz}$ flicker stimulation. Multifocal ERG showed substantially reduced central macula function in the left eye compared to the right eye (see fig 3 ). The flash VEP revealed good amplitudes in both eyes.

\section{DISCUSSION}

Complications associated with the removal of silicone oil are well documented and include re-detachment, hypotony, expulsive haemorrhage, and vitreous haemorrhage. ${ }^{6}$ We investigated three patients with "unexplained" visual loss following silicone oil removal.

Electrodiagnostic studies offer an objective method of assessing the visual pathway from the retina to the visual cortex. The results could be used to determine the location and possible nature of various dysfunctions along the pathway. It also provides information about the involvement of particular retinal cell types.

The PERG provides useful information in the distinction between optic nerve disease and macula disease in patients with poor central visual acuity. The N95 component reflects ganglion cell function where as the P50 component arises predominantly from the macula photoreceptors, horizontal, bipolar, and amacrine cells. ${ }^{7}$

In all three cases (especially cases 1 and 3 ) the PERG revealed increased latency and reduced amplitude of the P50 component with concomitant decreased amplitude of the N95 component in the affected eye. This is in keeping with abnormalities of the macula photoreceptors and middle layer function. In case 2, the PERG was reduced in both eyes which is presumably related to myopic degeneration. The mfERG showed a well defined hill of vision in the unaffected eye and a plateau effect in the affected eye indicating impaired

Table 1 Comparison of the electrophysiological responses between the normal and affected eye in all three cases

\begin{tabular}{|c|c|c|c|c|c|c|}
\hline \multirow[b]{2}{*}{ ERG test } & \multicolumn{2}{|l|}{ Case 1} & \multicolumn{2}{|l|}{ Case 2} & \multicolumn{2}{|l|}{ Case 3} \\
\hline & Affected eye & Normal eye & Affected eye & Normal eye & Affected eye & Normal eye \\
\hline $30 \mathrm{~Hz}$ flicker amplitude $(\mu \mathrm{V})$ & 85.0 & 106.0 & 65.0 & 90.0 & 50.0 & 80.0 \\
\hline $30 \mathrm{~Hz}$ flicker latency (ms) & 29.0 & 26.0 & 30.0 & 29.0 & 34.0 & 30.0 \\
\hline P50 $(\mu \mathrm{V})$ & 2.92 & 4.00 & 1.16 & 1.46 & 0.94 & 2.91 \\
\hline N95 $(\mu \mathrm{V})$ & 5.27 & 7.91 & 2.44 & 2.53 & 1.04 & 3.97 \\
\hline
\end{tabular}




\begin{tabular}{|l|l|l|l|l|l|l|}
\hline Fing & Amp.P1 [nivdeg] & Amp.P1[nv] & Amp.N1[nv] & PeT.N1[ms] & PeT.P1[ms] & Area [deg'] \\
\hline 1 & 97 & 0.65 & 0.21 & 20.6 & 38.2 & 7 \\
\hline 2 & 58 & 0.56 & 0.03 & 20.6 & 36.2 & 10 \\
\hline 3 & 53 & 0.74 & 0.32 & 16.7 & 34.3 & 14 \\
\hline 4 & 40 & 0.77 & 0.26 & 18.7 & 34.3 & 19 \\
\hline 5 & 22 & 0.57 & 0.04 & 18.6 & 35.3 & 26 \\
\hline & & & & & & \\
\hline
\end{tabular}

Figure 2 Multifocal ERG responses comparing affected right eye with unaffected fellow eye: case 1.

\section{Right eye}
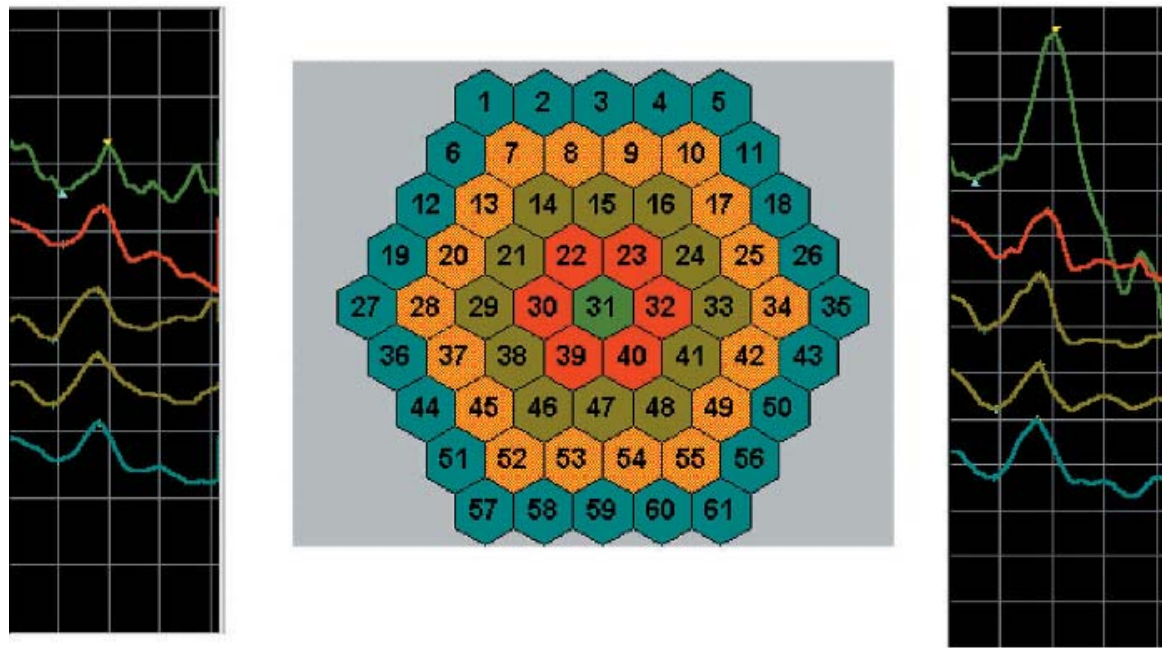

Left eye

\begin{tabular}{|c|c|c|c|c|c|c|}
\hline Fing & Amp.P1 [nY/deg'] & Amp.P $1[\mu v]$ & Amp.N1 $[\mu V]$ & $P \in T \cdot N 1[m s]$ & PeT.P1[ms] & Area [deg"] \\
\hline 1 & 234 & 1.58 & $\mid 0.02$ & 98 & 40.2 & 7 \\
\hline 2 & 52 & 0,50 & 0.09 & 18.6 & 37.2 & 10 \\
\hline 3 & 43 & 0.60 & 0.08 & 12.7 & 34.3 & 14 \\
\hline 4 & 25 & 0.48 & 0.14 & 17.6 & 34.3 & 19 \\
\hline 5 & 25 & 0.84 & 0.11 & 18.7 & 33.3 & 28 \\
\hline
\end{tabular}
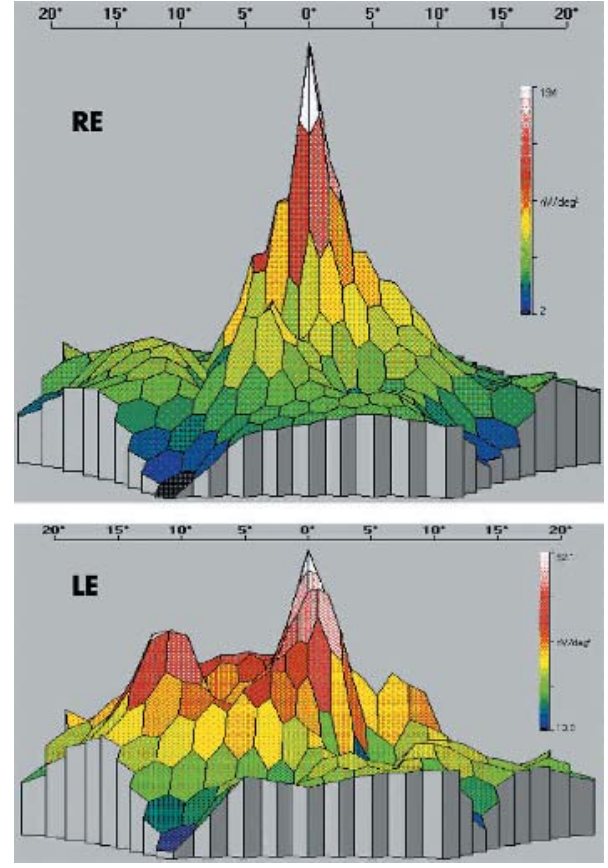

Figure 3 Multifocal responses comparing unaffected right eye with affected left eye: case 3. macula function in that eye (especially in cases 1 and 3). Additionally, it is the central ring that is affected more so than the outer ring, indicating a selective and severe damage to the fovea above and beyond what might be attributable to the original retinal damage (all three patients did not have any macula detachment at any stage and had good visual acuities following vitrectomies). The amplitudes of the four ERG flash tests were reduced in the affected eye compared to the unaffected. The flash VEPs were normal in all cases indicating that the visual pathways from the optic nerve onward remained intact.

Our results did not show any anatomical pathology to explain the drop in visual acuities. OCT was normal in each case. PERG, and particularly mfERG, does suggest that the dysfunction arises predominantly in the central part of the macula, involving mainly the outer and middle layers of the retina. The close temporal relation between oil removal and sudden visual loss suggests an event occurring during or soon after surgical intervention. The exact nature of the pathology remains obscure but is most likely related to physiochemical changes surrounding the event.

There is ample experimental evidence that Müller cells buffer the extracellular potassium ion concentration by siphoning excess $\mathrm{K}^{+}$ions into the vitreous. ${ }^{8-10}$ In normal eyes $\mathrm{K}^{+}$buffering is dependent on the vitreal sink and also retinal capillaries. $^{8}$

When vitreous is replaced by silicone oil efficient buffering of intraretinal increases of $\mathrm{K}^{+}$ions is achieved by the aqueous fluid between the oil and the retina. We can speculate that 
when the oil is removed there is a sudden dramatic physiochemical alteration in the aqueous milieu (potassium, glucose, depolarising transmitter molecules, and $\mathrm{pH}$ ) resulting in impaired buffering of $\mathrm{K}^{+}$ions. Such increases of $\mathrm{K}^{+}$ ions in the retina in turn, induce excitotoxicity and eventual neuronal cell damage, an effect similar to that described by Winter et al. ${ }^{11}$ It is also possible that an alteration in blood perfusion to the retina at the time of silicone oil removal may be a contributing factor. Another possible cause for visual loss could be the harmful effects of soluble growth factors and free radicals. Removing the oil, which acts as a physical barrier to these substances, allows more widespread dispersal and possibly damage to the macula as a result of accumulation at this site.

While retinal tamponade with intravitreal silicone oil is an effective method of treatment for complicated retinal detachments, its removal may be associated with the potential for retinal damage, from an as yet unidentified mechanism.

\section{ACKNOWLEDGEMENT}

We thank Richard Hagan of the Department of Clinical Engineering at the Royal Liverpool University Hospital, who carried out and provided the interpretation of the electrophysiological studies.

\section{Authors' affiliations}

S Cazabon, C Groenewald, I A Pearce, D Wong, St Paul's Eye Unit, Royal Liverpool University Hospital, Liverpool, UK
Correspondence to: Sunildath Cazabon, St Paul's Eye Unit, Royal Liverpool University Hospital, Prescott Street, Liverpool, L7 8XP, UK; jcazabon@hotmail.com

Accepted for publication 11 November 2004

\section{REFERENCES}

1 Azen SP, Scott IU, Flynn HW, et al. Silicone oil in the repair of complex retinal detachments. A prospective observational multicentre study. Ophthalmology 1998; 105: 1587-97.

2 Camacho H, Bajaire B, Mejia LF. Silicone oil in the management of giant retinal tears. Ann Ophthalmol 1992;24:45-9.

3 Alexandridis E. Silicone oil tamponade in the management of severe haemorrhagic detachment of the choroids and ciliary body after surgical trauma. Ophthalmologica 1990;200:189-93.

4 Federman JL, Schubert HD. Complications associated with the use of silicone oil in 150 eyes after retina-vitreous surgery. Ophthalmology 1988;95:870-5.

5 Lim JI, Enger C, Haller JA, et al. Improved visual results after surgical repair of cytomegalovirus-related retinal detachments. Ophthalmology 1994;101:264-9.

6 Casswell AG, Gregor ZT. Silicone oil removal. II. Operative and postoperative complications. Br J Ophthalmol 1987;71:898-902.

7 Holder GE. Significance of abnormal pattern electroretinography in anterior visual pathway dysfunction. Br J Ophthalmol 1987;71:166-71.

8 Newman EA. Distribution of potassium conductance in mammalian Müller (glial) cells: a comparative study. J Neurosci 1987;7:2423-32.

9 Newman EA, Frambach DA, Odette LL. Control of extracellular potassium levels by retinal glial cell $\mathrm{K}^{+}$siphoning. Science 1984;225:1174-5.

10 Oakley B, Katz BJ, Xu Z, et al. Spatial buffering of extracellular potassium by Müller (glial) cells in the toad retina. Exp Eye Res 1992;55:539-50.

11 Winter M, Eberhardt W, Scholz C, et al. Failure of potassium siphoning by Müller cells. A new hypothesis of perfluorocarbon liquid-induced retinopathy. Invest Ophthalmol Vis Sci 2000;41:256-61.

\section{bmjupdates+}

bmiupdates+ is a unique and free alerting service, designed to keep you up to date with the medical literature that is truly important to your practice.

bmjupdates + will alert you to important new research and will provide you with the best new evidence concerning important advances in health care, tailored to your medical interests and time demands.

Where does the information come from?

bmiupdates+ applies an expert critical appraisal filter to over 100 top medical journals A panel of over 2000 physicians find the few 'must read' studies for each area of clinical interest

Sign up to receive your tailored email alerts, searching access and more... www.bmjupdates.com 